\title{
Teenage Pregnancy and Its Associated Factors in Eastern Ethiopia: A Community-Based Study
}

This article was published in the following Dove Press journal:

International Journal of Women's Health

\section{Haymanot Mezmur (iC) \\ Nega Assefa (D) \\ Tadesse Alemayehu (D)}

College of Health and Medical Sciences, Haramaya University, Harar, Ethiopia
Correspondence: Haymanot Mezmur College of Health and Medical Sciences, Haramaya University, Po.box, 235, Harar, Ethiopia

Tel +25I-9l0-524422

Email mezmurhaymanot@yahoo.com
Background: Teenage pregnancy is a global issue raising concerns for all who are interested in the health and well-being of young women and their children. It carries major health and social issues with unique medical and psychosocial consequences for both adolescents and society in general. This study aimed at assessing the prevalence and factors associated with teenage pregnancy in eastern Ethiopia.

Methods: A community-based cross-sectional study was conducted. Multi-stage simple random sampling procedure was used to select 2258 female teenagers. Intervieweradministered questionnaire was used for data collection. Data were entered into EpiData and analyzed using stata software. The Poisson regression model with robust variance estimation was used to examine the association of the independent variable with teenage pregnancy. An adjusted prevalence ratio (APR) with 95\% confidence intervals (CI) was reported.

Results: The prevalence of teenage pregnancy was 30.2\% (95\% CI: 28.3, 32.1). Age 16-17 years old (APR $=7.05 ; 95 \%$ CI: 4.15,11.96), 17-18 years old $(A P R=9.85 ; 95 \%$ CI: $5.72,16.98$ ), not being in school ( $\mathrm{APR}=2.83$; $95 \% \mathrm{CI}: 1.93,4.16$ ), lack of formal education $(\mathrm{APR}=1.11 ; 95 \% \mathrm{CI}: 1.03,1.19)$, being married (APR=3.59; 95\% CI: 2.83,4.56), parental divorce (APR $=1.24 ; 95 \% \mathrm{CI}: 1.08,1.42$ ), having elder sister who had a history of teenage pregnancy $(\mathrm{APR}=1.11 ; 95 \% \mathrm{CI}: 1.02,1.21)$, and not knowing fertile period in menstrual cycle $(\mathrm{APR}=1.31 ; 95 \% \mathrm{CI}: 1.16,1.47)$ were independently associated with teenage pregnancy.

Conclusion: One in three teenagers had been pregnant. Age, not being in school, lack of formal education, being married, parental divorce, having an elder sister who had a history of teenage pregnancy, and not knowing fertile period during the menstrual cycles were the factors associated with teenage pregnancy. In Ethiopia, further efforts are required in the prevention of teenage pregnancy, keeping girls in school and strengthening the policy of delaying child marriage, particularly in rural areas.

Keywords: teenage pregnancy, associated factors, eastern Ethiopia

\section{Introduction}

According to the United Nations Children Fund (UNICEF), teenage pregnancy is defined as a pregnancy in girls within the ages of $13-19 .{ }^{1}$ It is a global problem and creates issues for all those concerned about young women and their children's health and well-being. ${ }^{2}$ Annually, about 21 million girls aged from 15 to 19 years in developing regions become pregnant. ${ }^{3}$ Around half of these pregnancies were unintended, and more than half ended in abortion, often in unsafe conditions. ${ }^{4}$

Sub-Saharan Africa recorded the highest prevalence of teenage pregnancy in the world in 2013. ${ }^{5,6}$ Teenage mothers accounted for more than half of all the births in this region. It is estimated that 101 births per 1000 women aged from 15 to 19 . This 
almost doubles the global average. ${ }^{5}$ From 15 countries that identified more than $30 \%$ of women giving birth before the age of 18 worldwide, 14 of them were found in SubSaharan Africa including Niger, Mozambique, Malawi, Uganda and Cameroon. ${ }^{6}$ The continent accounts for half of the world's burden of maternal, newborn and child deaths. $^{7}$

Teenage pregnancy should be one of the main issues in every healthcare system since early pregnancy can have harmful long-lasting implications on girls' physical, psychological, economic and social status ${ }^{8}$ and it is a concern from both human rights and public health perspectives. ${ }^{9}$ It is also a media-focal-point and a major issue irrespective of the teenager's marital status. ${ }^{10}$ The global maternal mortality ratio (MMR) in 2017 is estimated at 211 deaths per 100,000 live births, showing a reduction of $38 \%$ since $2000 .{ }^{11}$ One of the targets of sustainable development goals (SDGs 3.1) is ending preventable maternal deaths which aims for less than 70 maternal deaths per 100,000 live births globally by the year 2030. ${ }^{12}$ Preventing teenage pregnancy can help this goal since it is associated with poor maternal and child health outcomes and increased risks of dying during pregnancy. ${ }^{13}$

Teenage pregnancy can bring about major health and social problems with unique medical and psychosocial consequences for the adolescent and society at large. ${ }^{14}$ For instance, it prevents teenagers from achieving their full potential and enjoying their basic human rights. ${ }^{15}$ The effect of teenage pregnancy also adversely affects future earning potential and leads to lifelong poverty. ${ }^{16}$ Such negative impacts continue throughout a teenager's entire life and carry over to the next generation. ${ }^{17}$ Above all, it contributes to rapid population growth, especially in countries where contraceptive use is not well embraced and increases individual lifetime fertility. ${ }^{18}$ The contributing factors for teenage pregnancy are multiple and complex, ${ }^{19}$ categorized as socio-demographic, familial, cultural and reproductive behavior. Different literature reported that factors associated with teenage pregnancy include living in rural areas, not attending school, early marriage, lack of communication between parents and adolescents about sexual and reproductive health (SRH) issues, ${ }^{20}$ educational level of the teenagers, ${ }^{21}$ and family history of teenage pregnancy. ${ }^{22,23}$

Ethiopia is one of the African countries where the highest teenage pregnancies have been recorded. ${ }^{24}$ According to Ethiopian Demographic and Health Survey (EDHS), in 2016, the prevalence of teenage pregnancy was $16 \%$. The prevalence was common in rural than in urban areas, (15\%) and (5\%), respectively. ${ }^{25}$ Most of the adolescent girls in rural areas of Ethiopia become pregnant every year outside marriage. ${ }^{26}$ Teen pregnancy was also highly prevalent among those without any education $(28 \%)$, primary level education $(12 \%)$ and secondary level education $(3 \%){ }^{25}$

There are gaps in research and publication regarding teenage pregnancy in Ethiopia. The existing teenage pregnancy research fails to clearly breakdown factors contributing to teenage pregnancy into specific categories, especially in rural areas, and most of the works are institutional-based and small-scale studies. ${ }^{27-29}$ Factors contributing to teenage pregnancy are not well studied in Ethiopia. Therefore, this community-based study aimed at assessing the prevalence and factors associated with teenage pregnancy in eastern Ethiopia.

\section{Methods}

\section{Study Design and Setting}

A community-based cross-sectional study was carried out from January to March 2020 in Kersa Health and Demographic Surveillance System (HDSS), which is in Kersa District, East Hararghe Zone, Oromia Regional State. The site consists of 138,354 populations. The inhabitants of the study site make their living mainly on farming. The District contained a total number of 38 Kebeles (the smallest administrative unit in Ethiopia) of which 24 Kebeles were included in Kersa HDSS. There are 19 health posts and 6 health centers on Kersa HDSS site. All of the Kebeles have 2-3 health extension workers. ${ }^{30}$

\section{Population and Sampling Technique}

Female teenagers 13-19 years of age who had resided in the study site for at least 6 months were included in the study. The sample size was determined with an assumption of $95 \%$ significance level, power $90 \%$, and proportion of teenage pregnancy among teenagers whose parents married was $85.7 \%$, which was taken from a previous study. ${ }^{29}$ A design effect of two and $10 \%$ non-response rate resulted in the final sample size of 2258. Multi-stage simple random sampling was used to select the representative samples. Out of the 24 Kebeles under the Kersa HDSS site, 12 were selected through lottery method. Proportional to the size of the study population in each individual Kebele was used to enroll the study participants. Following the sampling frame was obtained from Kersa HDSS database, the 
study subject was randomly selected from the designated Kebeles. Finally, the household IDs of the selected teenagers were retrieved from the database.

\section{Data Collection Tools, Quality Assurance and Pretest}

A structured-interviewer-administered questionnaire was used for data collection. The questionnaire was adapted from the WHO (Illustrative-questionnaire for interview survey with young people). ${ }^{31}$ The original questionnaire was prepared in English and then translated into a local language (Afan Oromo) for data collection. Then, the Afan Oromo version was back-translated into English to check for consistency. The translations were performed by experts who were competent in both English and Afan Oromo. Pretest was done in a comparable setting. Based on the results, the necessary amendment was made to refine the questionnaire.

Data were collected by trained and experienced 16 female data collectors and 4 field supervisors. Before deploying data collectors to the field for actual data collection, training was given to the data collectors and the field supervisors for 3 days on interviewing techniques and field procedures, and thorough discussion was made on the data collection tools. Fieldwork and ethical issue manuals were used during training and given to the data collectors and supervisors to be used as a reference during the actual data collection. Completeness, accuracy, and clarity of the collected data were checked carefully by supervisors daily.

\section{Ethical Approval}

This study was conducted following the Declaration of Helsinki. Ethical approval was obtained from the Institutional Health Research Ethics Review Committee of Haramaya University, College of Health and Medical Sciences before the commencement of data collection (Ref.no. IHRERC/106/2020). Besides, written informed consent was obtained from each study participant, and assent was obtained from girls below the age of 18 before the interview. Parental or legal guardians of girls below the legal age of 18 were given written informed consent. To ensure confidentiality, the names of the participants were not requested and recorded.

\section{Variables and Measurement}

The outcome variable of this study was teenage pregnancy. Teenage pregnancy was assessed based on teenager response whether she had ever been pregnant or not, including pregnancy ending in abortion, stillbirth, live birth and pregnancy during the time of the survey. Then, the response was categorized as 0 "no", 1 "yes".

The independent variables included socio-demographic characteristics, family-related variables and reproductive behaviors. Socio-demographic variables that comprise age in completed years were categorized as 13-14 years old, 15-17 years old, 18-19 years old; school attendance was coded as in school or not in school; educational level coded as no formal education or have formal education; and marital status coded as never married or ever married.

The family-related variables were coded as follows: The wealth distribution was calculated using Principal Component Analysis (PCA) method. Twenty-three items were included in the analysis. Seven components were taken out based on Eigenvalues $>1$ and examination of scree plots. The components were categorized into five quantiles and coded as lowest, second, middle, fourth or highest; parental marital status coded as married, widowed or divorced; type of family raised by coded as both biological parents, one biological parent or neither biological parent; and elder sister history of teenage pregnancy coded as yes or no.

Finally, reproductive health-related variables were coded as follows: discussion between parents and teenager on reproductive health issues was coded as yes or no; getting counselling and guidance from parents regarding reproductive health coded as yes or no; received sex education at school coded as yes or no; knowing fertile period during menstrual cycle coded as yes or no; and knowing the exact time to take emergency contraceptive coded as yes or no.

\section{Data Analysis}

Data completeness and consistency were checked at the field level and coded. Double data entry using EpiData version 3.1 was performed to check consistency and exported to stata version 14 for analysis. The frequency distribution was calculated using descriptive statistics. Proportions with 95\% CI were used to calculate the prevalence of teenage pregnancy. Poisson regression analysis model with robust variance estimation was used to examine the association of the independent variables with teenage pregnancy.

Poisson regression model is an example of a generalized linear model used to directly estimate the prevalence ratio in cross-sectional studies. The Poisson regression with robust variance does not have any 
convergence difficulty while applying to binomial data. ${ }^{32}$ The prevalence ratio can be overestimated by the odds ratio when the outcome variable is frequent, usually with a prevalence greater than $10 \% .{ }^{33}$

To identify the candidate variables for multivariable model building, bivariate analysis was carried out and those variables with a p-value $<0.25$ were considered. To assess the association of independent variables with teenage pregnancy, four regression models were built. Before the final model, three models were built to assess the effect of variables.

Variance inflation factor (VIFs) and correlation matrix for the regression coefficients were used to detect the presence of multicollinearity between independent variables. Respondent occupation was omitted from the final model due to multicollinearity with school attendance. The final model was selected based on Akaike's and Bayesian information criterion. Model fitness was assessed using Pearson Chi-square and Hosmer-Lemeshow goodness-of-fit test. Model I included teenagers' socio-demographic variables; Model II comprised familial variables; Model III contained reproductive characteristic variables, and Model IV was built using socio-demographic, familial and reproductive variables. Adjusted prevalence ratios (APRs) with 95\% CI were used to report the results. Statistical level of significance was declared at $\mathrm{p}$ value $<0.05$.

\section{Results}

\section{Socio-Demographic Characteristics of Study Participants}

A total of 2258 female teenagers participated in this study. Their age ranges from 13 to 19 years with the mean and standard deviation (SD) of $16.1( \pm 2)$. Out of the total participants, $2180(96.6 \%)$ were belonging to Islam religion and $1041(46 \%)$ of them were attending their education in public schools. Around $1583(70 \%)$ had formal education. Two-third of teenagers $(67.6 \%)$ were never married and 731 (32\%) were married during survey time. Among married teenagers, the mean age at marriage was $15.7( \pm 1.2)$ (Table 1).

\section{Family Level and Reproductive Health Characteristics}

Two thousand twelve (89.1\%) of the teenagers' parents were married. Around 1977 (87.5\%) were raised by both biological parents and $781(34.6 \%)$ reported have elder sisters who had a history of teenage pregnancy. The
Table I Socio-Demographic Characteristics of the Study Participants Living in Kersa HDSS, Eastern Ethiopia, 2020

\begin{tabular}{|c|c|c|}
\hline Variables & Number $(\mathrm{N}=2258)$ & Percent \\
\hline \multicolumn{3}{|l|}{ Age } \\
\hline $13-15$ years & 981 & 43.4 \\
\hline $16-17$ years & 553 & 24.5 \\
\hline $18-19$ years & 724 & 32.1 \\
\hline \multicolumn{3}{|l|}{ Religion } \\
\hline Muslim & 2180 & 96.6 \\
\hline Others $^{\mathrm{a}}$ & 78 & 3.4 \\
\hline \multicolumn{3}{|l|}{ Ethnicity } \\
\hline Oromo & 2195 & 97.2 \\
\hline Others $^{b}$ & 63 & 2.8 \\
\hline \multicolumn{3}{|l|}{ School attendance } \\
\hline In school & 1041 & 46.1 \\
\hline Not in school & 1217 & 53.9 \\
\hline \multicolumn{3}{|l|}{ Educational level } \\
\hline No formal education & 675 & 29.9 \\
\hline Have a formal education & 1583 & 70.1 \\
\hline \multicolumn{3}{|l|}{ Occupational status } \\
\hline Student & 1028 & 45.5 \\
\hline House wife & 704 & 31.2 \\
\hline Self employed & 54 & 2.4 \\
\hline Unemployed & 472 & 20.9 \\
\hline \multicolumn{3}{|l|}{ Marital status } \\
\hline Never married & 1527 & 67.6 \\
\hline Ever married & 731 & 32.4 \\
\hline \multicolumn{3}{|l|}{ Age at Ist marriage ( $\mathrm{N}=73 \mathrm{I})$} \\
\hline 13-14years & 95 & 13.0 \\
\hline I5-17years & 582 & 79.6 \\
\hline $18-19$ years & 54 & 7.4 \\
\hline
\end{tabular}

Notes: ${ }^{a}$ Orthodox and Protestant. ${ }^{\mathrm{b}} \mathrm{Amhara}$ and Gurage.

majority, 2038 (90.3\%) of teenagers did not communicate with their parents regarding reproductive health $(\mathrm{RH})$ issues. Likewise, $1982(87.8 \%)$ of the teenagers had not received any counseling from their families regarding $\mathrm{RH}$ issues, whereas about 542 (24\%) of them received sexual education at school. Only, $389(17.2 \%)$ of the teenagers knew the fertile period in the menstrual cycle, and 193 $(8.6 \%)$ knew the appropriate time when emergency contraceptives should be taken (Table 2).

\section{Prevalence of Teenage Pregnancy}

The prevalence of teenage pregnancy in the study was $30.2 \%$ (95\% CI: 28.3,32.1). A higher prevalence of 
Table 2 Family Level and Reproductive Health Characteristics of the Study Participants Living in Kersa HDSS, Eastern Ethiopia, 2020

\begin{tabular}{|c|c|c|}
\hline Variables & $\begin{array}{l}\text { Number } \\
(\mathbf{N}=\mathbf{2 2 5 8})\end{array}$ & Percent \\
\hline \multicolumn{3}{|l|}{ Wealth index } \\
\hline Lowest & 477 & 21.1 \\
\hline Second & 445 & 19.7 \\
\hline Middle & 433 & 19.2 \\
\hline Fourth & 452 & 20.0 \\
\hline Highest & 451 & 20.0 \\
\hline \multicolumn{3}{|l|}{ Educational status of the mother } \\
\hline No formal education & 2082 & 92.2 \\
\hline Have a formal education & 176 & 7.8 \\
\hline \multicolumn{3}{|l|}{ Occupational status of the mother } \\
\hline Housewife & 2127 & 94.2 \\
\hline Others $^{c}$ & 131 & 5.8 \\
\hline \multicolumn{3}{|l|}{ Educational status of the father } \\
\hline No formal education & 1835 & 81.3 \\
\hline Have a formal education & 423 & 18.7 \\
\hline \multicolumn{3}{|l|}{ Occupational status of the father } \\
\hline Farmer & 1993 & 88.3 \\
\hline Others & 265 & 11.7 \\
\hline \multicolumn{3}{|l|}{ Parent marital status } \\
\hline Married & 2012 & 89.1 \\
\hline Widowed & 79 & 3.5 \\
\hline Divorced & 167 & 7.4 \\
\hline \multicolumn{3}{|l|}{ Type of parent raised } \\
\hline Both biological parents & 1977 & 87.5 \\
\hline Either of biological parent & 126 & 5.6 \\
\hline Guardian/adoptive parents & 155 & 6.9 \\
\hline \multicolumn{3}{|l|}{ Sisters history of teenage pregnancy } \\
\hline Yes & 781 & 34.6 \\
\hline No & 1477 & 65.4 \\
\hline \multicolumn{3}{|l|}{$\begin{array}{l}\text { Communication with parents on } \mathrm{RH} \\
\text { issues }\end{array}$} \\
\hline Yes & 220 & 9.7 \\
\hline No & 2038 & 90.3 \\
\hline \multicolumn{3}{|l|}{ Get family counselling } \\
\hline Yes & 276 & 12.2 \\
\hline No & 1982 & 87.8 \\
\hline \multicolumn{3}{|l|}{ Received sex education at school } \\
\hline Yes & 542 & 24.0 \\
\hline No & 1716 & 76.0 \\
\hline \multicolumn{3}{|l|}{ Knew fertile period in menstrual cycle } \\
\hline Yes & 389 & 17.2 \\
\hline No & 1869 & 82.8 \\
\hline
\end{tabular}

(Continued)
Table 2 (Continued).

\begin{tabular}{|l|l|l|}
\hline Variables & $\begin{array}{l}\text { Number } \\
(\mathbf{N = 2 2 5 8 )}\end{array}$ & Percent \\
\hline $\begin{array}{l}\text { Knew the accurate time to take } \\
\text { emergency contraceptive(EC) }\end{array}$ & \\
Yes & 193 & 8.6 \\
No & 2065 & 91.4 \\
\hline
\end{tabular}

Note: 'In occupation type includes government employee, self-employ, unemployed.

teenage pregnancy was among housewife teenagers compared to students [84\% (95\% CI: $80.7,86.2)$ versus $2.2 \%$ (95\% CI: 1.5,3.3)]. Moreover, the prevalence of teenage pregnancy among ever married teenagers was [82\% $(95 \%$ CI: 78.7, 84.3)] compared to never married teenagers [5.6\% (95\% CI: 4.5,6.8)]. Also, the prevalence observed in the age range of $18-19$ years old was $[70.1 \%$ (95\% CI: $66.7,73.4)]$ compared to teenagers with the age range of $13-15$ years old $[1.6 \%(95 \% \mathrm{CI}: 1.0,1.6)]$. The prevalence of teenage pregnancy was high among teenagers whose parents were divorced compared to married [53\% (95\% CI: 45, 60.2) versus 28\% (95\% CI: 26.1,30)] (Table 3).

\section{Factors Associated with Teenage Pregnancy}

After controlling for confounding factors, the prevalence ratio of teenage pregnancy among the teenagers who were 16-17 years old was 7.05 times higher than that of teenagers who were $13-14$ years old (APR=7.05; 95\% CI: 4.15,11.96). The ratio was also 9.85 higher among teenagers with the age range of 18-19 compared to teenagers with the age range of 13-14 (APR=9.85; 95\% CI :5.72,16.98).

The prevalence ratio of teenage pregnancy among teenagers who were being out of school was 2.83 times higher than those being in school $(\mathrm{APR}=2.83$; 95\% CI: 1.93,4.16). The prevalence of TP was also $11 \%$ higher among teenagers with no formal education compared to those who had formal education $(\mathrm{APR}=1.11 ; 95 \% \mathrm{CI}: 1.03,1.19)$. The prevalence ratio of teenage pregnancy among married teens was 3.59 times higher than those who never married $(\mathrm{APR}=3.59$; 95\% CI: 2.83,4.56). Again, the prevalence ratio of TP among teenagers from divorced parents was 1.24 times higher than married parents $(\mathrm{APR}=1.24 ; 95 \% \mathrm{CI}$ : 1.08,1.42). Similarly, having an elder sister who had a history of teenage pregnancy was significantly asso- 
Table 3 Prevalence of Different Contributing Factors for Teenage Pregnancy Among Study Participants Living in Kersa HDSS, Eastern Ethiopia, 2020

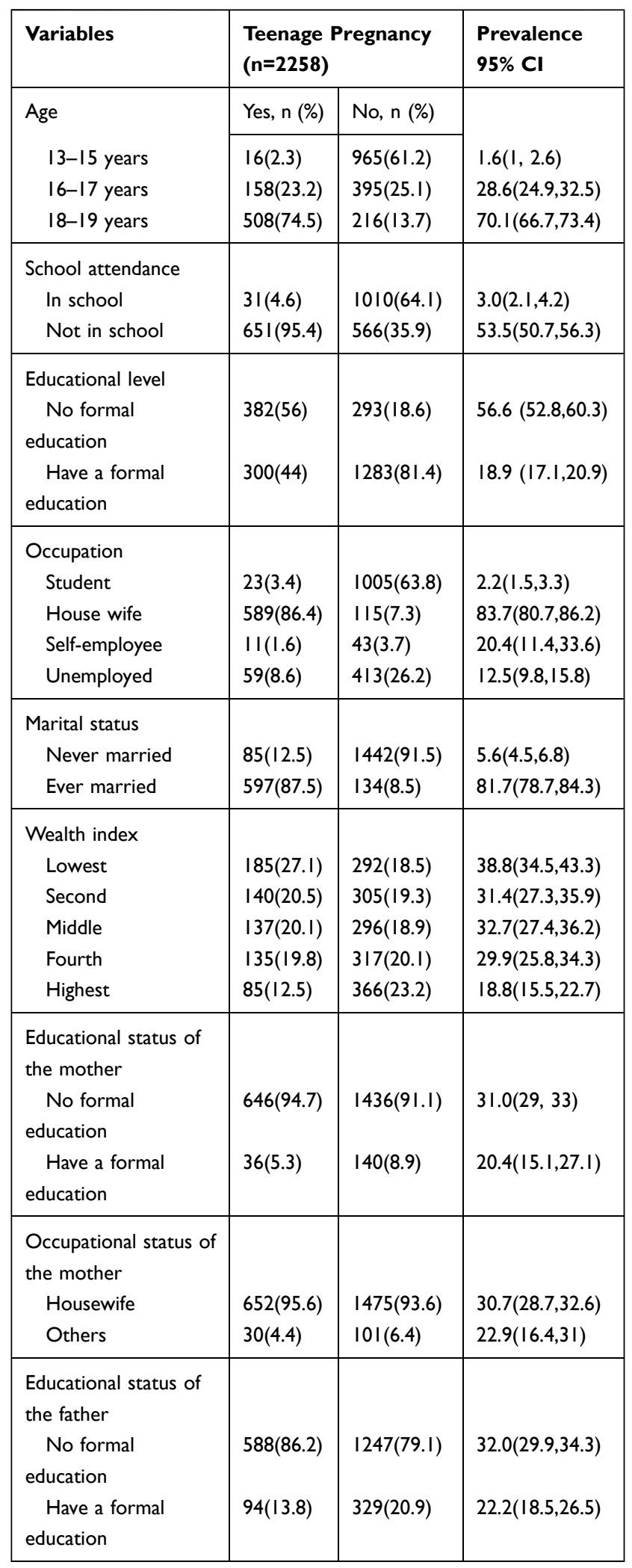

(Continued)
Table 3 (Continued).

\begin{tabular}{|c|c|c|c|}
\hline Variables & $\begin{array}{l}\text { Teenage } \\
(n=2258)\end{array}$ & regnancy & $\begin{array}{l}\text { Prevalence } \\
95 \% \mathrm{Cl}\end{array}$ \\
\hline $\begin{array}{l}\text { Occupational status of } \\
\text { the father } \\
\text { Farmer } \\
\text { Others }\end{array}$ & $\begin{array}{l}610(89.4) \\
72(10.6)\end{array}$ & $\begin{array}{l}1383(87.8) \\
193(\mid 2.2)\end{array}$ & $\begin{array}{l}30.6(28.6,32.7) \\
27.2(22.1,32.9)\end{array}$ \\
\hline $\begin{array}{l}\text { Parental marital status } \\
\text { Married } \\
\text { Widowed } \\
\text { Divorced }\end{array}$ & $\begin{array}{l}563(82.6) \\
31(4.6) \\
88(12.9)\end{array}$ & $\begin{array}{l}1449(9 \mid .9) \\
48(3.1) \\
79(5.0)\end{array}$ & $\begin{array}{l}27.9(26.1,30) \\
39.2(28.9,50.6) \\
52.7(45,60.2)\end{array}$ \\
\hline $\begin{array}{l}\text { Type of Family raised } \\
\text { Both biological } \\
\text { parents } \\
\text { Either of bio. } \\
\text { Parents } \\
\text { Neither of bio. } \\
\text { Parents }\end{array}$ & $\begin{array}{l}552(80.9) \\
50(7.3) \\
80(11.7)\end{array}$ & $\begin{array}{l}1425(90.4) \\
76(4.8) \\
75(4.8)\end{array}$ & $\begin{array}{l}27.9(25.9,29.9) \\
39.7(31.4,48.6) \\
51.6(43.7,59.5)\end{array}$ \\
\hline $\begin{array}{l}\text { Elder sister history of } \\
\text { teenage pregnancy } \\
\text { Yes } \\
\text { No }\end{array}$ & $\begin{array}{l}396(58.1) \\
386(41.9)\end{array}$ & $\begin{array}{l}385(24.4) \\
1191(75.6)\end{array}$ & $\begin{array}{l}50.7(47.2,54.2) \\
19.4(|7.4,2| .5)\end{array}$ \\
\hline $\begin{array}{l}\text { Discussion with } \\
\text { parents on } \mathrm{RH} \text { issues } \\
\text { Yes } \\
\text { No }\end{array}$ & $\begin{array}{l}20(2.9) \\
662(97.1)\end{array}$ & $\begin{array}{l}200(12.7) \\
1376(87.3)\end{array}$ & $\begin{array}{l}9.0(5.9,13.7) \\
32.5(30.5,34.5)\end{array}$ \\
\hline $\begin{array}{l}\text { Get family counselling } \\
\text { Yes } \\
\text { No }\end{array}$ & $\begin{array}{l}25(3.7) \\
657(96.3)\end{array}$ & $\begin{array}{l}25 \mid(\mid 5.9) \\
1325(84.1)\end{array}$ & $\begin{array}{l}9.0(6.2,13.1) \\
33.1(31.1,35.3)\end{array}$ \\
\hline $\begin{array}{l}\text { Received sex } \\
\text { education at school } \\
\text { Yes } \\
\text { No }\end{array}$ & $\begin{array}{l}61(8.9) \\
621(91.1)\end{array}$ & $\begin{array}{l}48 I(30.5) \\
1095(69.5)\end{array}$ & $\begin{array}{l}\text { II.3(8.8, |4.2) } \\
36.2(33.9,38.5)\end{array}$ \\
\hline $\begin{array}{l}\text { Knew fertile period in } \\
\text { menstrual cycle } \\
\text { Yes } \\
\text { No }\end{array}$ & $\begin{array}{l}100(14.7) \\
582(85.3)\end{array}$ & $\begin{array}{l}289(18.3) \\
1287(8 \mid .7)\end{array}$ & $\begin{array}{l}25.7(21.6, \\
30.3) \\
31.1(29.1,33.3)\end{array}$ \\
\hline $\begin{array}{l}\text { Knew the accurate } \\
\text { time to take EC } \\
\text { Yes } \\
\text { No }\end{array}$ & $\begin{array}{l}27(4.0) \\
655(95.0)\end{array}$ & $\begin{array}{l}166(10.5) \\
1410(89.5)\end{array}$ & $\begin{array}{l}\text { I3.9(9.7,19.7) } \\
31.7(29.7,33.8)\end{array}$ \\
\hline Ever had pregnancy & $682(30.2)$ & $1576(69.8)$ & $30.2(28.3,32.1)$ \\
\hline
\end{tabular}

ciated with teenage pregnancy $(\mathrm{APR}=1.11 ; 95 \% \mathrm{CI}$ : $1.02,1.21)$ and the prevalence of teenage pregnancy was $31 \%$ higher among teenagers who did not know 
Table 4 Factors Associated with Teenage Pregnancy Among Study Participants Living in Kersa HDSS Eastern Ethiopia, 2020

\begin{tabular}{|c|c|c|c|c|c|}
\hline Variables & CPR $(95 \% \mathrm{Cl})$ & $\begin{array}{l}\text { Model } \\
\text { I (Demographic } \\
\text { Factors) APR } \\
(95 \% \mathrm{CI})\end{array}$ & $\begin{array}{l}\text { Model II } \\
\text { (Family-Related } \\
\text { Factors) APR } \\
(95 \% \mathrm{CI})\end{array}$ & $\begin{array}{l}\text { Model III } \\
\text { (Reproductive } \\
\text { Characteristics } \\
\text { Factors) APR (95\% } \\
\text { Cl) }\end{array}$ & $\begin{array}{l}\text { Final Model } \\
\text { APR }(95 \% \mathrm{Cl})\end{array}$ \\
\hline $\begin{array}{l}\text { Age } \\
\qquad \begin{array}{l}13-15 \text { Years } \\
16-17 \text { years } \\
18-19 \text { years }\end{array}\end{array}$ & $\begin{array}{l}\text { I } \\
17.52(10.59,28.99)^{*} \\
43.02(26.39,70.11)^{*}\end{array}$ & $\begin{array}{l}\text { 6.8(4.05, II.56)* } \\
9.57(5.58,16.40)^{*}\end{array}$ & & & $\begin{array}{l}\text { I } \\
7.05(4.15,11.96)^{*} \\
9.85(5.72,16.98)^{*}\end{array}$ \\
\hline $\begin{array}{l}\text { Schooling attendance } \\
\text { In school } \\
\text { Not in school }\end{array}$ & $\begin{array}{l}\text { I } \\
I .96(\mid 2.65,25.5 I)^{*}\end{array}$ & $\begin{array}{l}\text { I } \\
3.48(2.39,5.07)^{*}\end{array}$ & & & $\begin{array}{l}\text { I } \\
2.83(1.93,4.16)^{*}\end{array}$ \\
\hline $\begin{array}{l}\text { Educational level } \\
\text { No formal education } \\
\text { Have a formal } \\
\text { education }\end{array}$ & $\begin{array}{l}2.98(2.64,3.37)^{*} \\
\text { I }\end{array}$ & $\begin{array}{l}1.11(1.03,1.19)^{*} \\
I\end{array}$ & & & $\begin{array}{l}\mathrm{I} . \mathrm{II}(1.03,1.19)^{*} \\
\mathrm{I}\end{array}$ \\
\hline $\begin{array}{l}\text { Marital status } \\
\text { Never married } \\
\text { Ever married }\end{array}$ & $\begin{array}{l}\text { I } \\
\mid 4.67(|1.89,| 8.09)^{*}\end{array}$ & $\begin{array}{l}\text { I } \\
3.60(2.81,4.63)^{*}\end{array}$ & & & $\begin{array}{l}\text { I } \\
3.59(2.83,4.56)^{*}\end{array}$ \\
\hline $\begin{array}{l}\text { Wealth index } \\
\text { Lowest } \\
\text { Second } \\
\text { Middle } \\
\text { Fourth } \\
\text { Highest }\end{array}$ & $\begin{array}{l}2.06(1.65,2.57)^{*} \\
1.67(1.32,2.11)^{*} \\
168(1.33,2.13)^{*} \\
1.58(1.25,2.01)^{*} \\
1\end{array}$ & & $\begin{array}{l}1.69(1.36,2.08)^{*} \\
1.59(1.28,1.99)^{*} \\
1.49(1.19,1.88)^{*} \\
1.42(1.14,1.78)^{*} \\
\text { I }\end{array}$ & & $\begin{array}{l}\text { I.I4(0.99,I.32) } \\
1.13(0.97,1.31) \\
1.11(0.96,1.29) \\
1.09(0.94,1.27) \\
\text { I }\end{array}$ \\
\hline $\begin{array}{l}\text { Educational status of the } \\
\text { mother } \\
\text { No formal education } \\
\text { Have a formal } \\
\text { education }\end{array}$ & $1.5 \mathrm{I}(\mathrm{I} .12,2.04)^{*}$ & & $\begin{array}{l}I .21(0.89,1.65) \\
I\end{array}$ & & $1.03(0.84,1.26)$ \\
\hline $\begin{array}{l}\text { Occupational status of the } \\
\text { mother } \\
\text { *Other occupation } \\
\text { Housewife }\end{array}$ & $\begin{array}{l}\text { I } \\
\text { I.33(0.97, I.84) }\end{array}$ & & $1.31(0.97,1.77)^{*}$ & & $\begin{array}{l}1 \\
0.96(0.76,1.21)\end{array}$ \\
\hline $\begin{array}{l}\text { Educational status of the } \\
\text { father } \\
\text { No formal education } \\
\text { Have a formal } \\
\text { education }\end{array}$ & $1.44(I .19,1.74)^{*}$ & & $1.22(1.00,1.48)^{*}$ & & $0.98(0.87,1.12)$ \\
\hline $\begin{array}{l}\text { Parental marital status } \\
\text { Married } \\
\text { Widowed } \\
\text { Divorced }\end{array}$ & $\begin{array}{l}1.40(1.05,1.86)^{*} \\
1.88(1.60,2.21)^{*}\end{array}$ & & $\begin{array}{l}\text { I } \\
1.43(1.11,1.84)^{*} \\
1.68(1.39,2.01)^{*}\end{array}$ & & $\begin{array}{l}\text { I } \\
1.01(0.78,1.30) \\
1.24(1.08,1.42)^{*}\end{array}$ \\
\hline $\begin{array}{l}\text { Type of Family raised } \\
\text { Both biological parents }\end{array}$ & I & & I & & I \\
\hline
\end{tabular}

(Continued) 
Table 4 (Continued).

\begin{tabular}{|c|c|c|c|c|c|}
\hline Variables & CPR $(95 \% \mathrm{CI})$ & $\begin{array}{l}\text { Model } \\
\text { I (Demographic } \\
\text { Factors) APR } \\
(95 \% \mathrm{CI})\end{array}$ & $\begin{array}{l}\text { Model II } \\
\text { (Family-Related } \\
\text { Factors) APR } \\
(95 \% \mathrm{CI})\end{array}$ & $\begin{array}{l}\text { Model III } \\
\text { (Reproductive } \\
\text { Characteristics } \\
\text { Factors) APR (95\% } \\
\text { Cl) }\end{array}$ & $\begin{array}{l}\text { Final Model } \\
\text { APR }(95 \% \mathrm{CI})\end{array}$ \\
\hline $\begin{array}{l}\text { Either of bio. Parents } \\
\text { Neither of bio. Parents }\end{array}$ & $\begin{array}{l}1.42(I .13,1.78)^{*} \\
1.84(1.56,2.18)^{*}\end{array}$ & & $\begin{array}{l}0.81(0.65,1.03)^{*} \\
1.33(1.13,1.57)^{*}\end{array}$ & & $\begin{array}{l}I . I I(0.91, I .35) \\
I .09(0.95, I .26)\end{array}$ \\
\hline $\begin{array}{l}\text { Elder sister history of } \\
\text { teenage pregnancy } \\
\text { Yes } \\
\text { No }\end{array}$ & $2.62(2.31,2.97)^{*}$ & & $2.46(2.17,2.79)^{*}$ & & $\mathrm{I} . \mathrm{II}(\mathrm{I} .02, \mathrm{I} .2 \mathrm{I})^{*}$ \\
\hline $\begin{array}{l}\text { Discussion with parents } \\
\text { on } \mathrm{RH} \text { issues } \\
\text { Yes } \\
\text { No }\end{array}$ & $0.28(0.18,0.43)^{*}$ & & & $0.59(0.38,0.90)^{*}$ & $0.77(0.57,1.05)$ \\
\hline $\begin{array}{l}\text { Get family counselling } \\
\text { Yes } \\
\text { No }\end{array}$ & $\begin{array}{l}0.27(0.18,0.39)^{*} \\
\mathrm{I}\end{array}$ & & & $\begin{array}{l}0.57(0.38,0.84)^{*} \\
I\end{array}$ & $\begin{array}{l}\mathrm{I} .02(0.75, \mathrm{I} .38) \\
\mathrm{I}\end{array}$ \\
\hline $\begin{array}{l}\text { Received Sex education at } \\
\text { school } \\
\text { Yes } \\
\text { No }\end{array}$ & $\begin{array}{l}0.3 \mathrm{I}(0.24,0.39)^{*} \\
\mathrm{I}\end{array}$ & & & $\begin{array}{l}0.4 \mathrm{I}(0.32,0.53)^{*} \\
\mathrm{I}\end{array}$ & $0.96(0.82, I .13)$ \\
\hline $\begin{array}{l}\text { Knew fertile period in } \\
\text { menstrual cycle } \\
\text { Yes } \\
\text { No }\end{array}$ & $1.21(1.01,1.45)^{*}$ & & & $\begin{array}{l}\text { I } \\
0.98(0.83,1.17)\end{array}$ & $1.31(1.16,1.47)^{*}$ \\
\hline $\begin{array}{l}\text { Knew the accurate time } \\
\text { to take EC } \\
\text { Yes } \\
\text { No }\end{array}$ & $\begin{array}{l}0.44(0.31,0.63)^{*} \\
\text { I }\end{array}$ & & & $0.64(0.45,0.91)^{*}$ & $0.81(0.59,1.09)$ \\
\hline
\end{tabular}

Notes: ${ }^{*} \mathrm{p}<0.2$ in crude prevalence ratio (CPR); ${ }^{*} \mathrm{p}<0.05$ in adjusted prevalence ratio (APR).

the fertile period in menstrual cycle $(\mathrm{APR}=1.31 ; 95 \%$ CI: 1.16,1.47) (Table 4).

\section{Discussion}

This study assessed the prevalence and factors associated with teenage pregnancy. Accordingly, one in three teenagers experienced pregnancy. Factors such as age, not being in school, lack of formal education, being married, parental divorce, having an elder sister who had a history of teenage pregnancy, and not knowing fertile period in the menstrual cycles were all independently associated with teenage pregnancy.

In this study, the prevalence of teenage pregnancy was $30.2 \%$ which is consistent with results found by Habitu et al in Northeast Ethiopia ${ }^{29}$ that indicated a prevalence rate of $28.6 \%$ and a study conducted by Okereke in Kenya ${ }^{34}$ which reported a prevalence rate of $31.6 \%$. On the other hand, a study conducted by Nwosu in the Abia State of Nigeria reported a prevalence of $49 \%,{ }^{35}$ which is higher than the rate found in this study. The discrepancy could be explained in light of the high rate of unemployed participants in the study conducted in Nigerian $(75.8 \%)$. Results revealed that around 54\% of the pregnancies occurred among unemployed teenagers. Whereas in this study, the occurrence of pregnancy in unemployed participants accounts for only $12 \%$. This may be due to contraceptive use was higher among employed women than that of unemployed ones, as economic role gives them more advantage and more control over important decisions. ${ }^{36}$ On the other hand, 
the cost of contraception services and methods are potential barriers to the adolescent. In some countries, contraceptive is provided at no cost and may resolve the financial barrier, but does not assurance high rates of utilization. $^{37}$

The prevalence of teenage pregnancy in this study $(30.2 \%)$ is higher than that of the Ethiopian national report in 2016, which was $13 \% .^{25}$ The inconsistency between these two estimates may be due to the national study included a larger sample, both urban and rural areas. In contrast, this study was conducted in a rural district of Oromia Region with a high prevalence of early marriage. About $32.4 \%$ of the participants were married, among which $79.6 \%$ were married before the age of 18 , with the mean age of first marriage at 15.7 years. As a result, all these factors may contribute to the high prevalence of teenage pregnancy in this area.

The prevalence ratio of teenage pregnancy increased as age increased. Accordingly, the percentage of adolescent pregnancy among teenagers of 13-14 years old was $2.4 \%$ compared to those between 18 and 19 years old (74.5\%). Similarly, $69.8 \%$ of teenagers with $18-19$ years were married compared to $5.1 \%$ in the $13-15$ years old. This may be explained as age increases the probability of sexual intercourse and marriage also increases; as a result, the risk of exposure to pregnancy and childbearing also increases. $^{38}$ This finding is consistent with studies conducted by Were in Kenya ${ }^{39}$ and Ayele et al in Ethiopia ${ }^{40}$ which indicated that as the age of the adolescent increased, the odds of an adolescent pregnancy also increased.

The prevalence ratio of teenage pregnancy was 2.83 times higher among teenagers currently not attending school than those attending school. This finding is supported by studies carried out in South Africa, ${ }^{41}$ and Malawi $^{42}$ which showed that being out of school was associated with pregnancy. Literature suggests that the attendance of formal education creates behavioral change. ${ }^{43}$ School attendance may provide periods of structure and supervision which may decrease the chances to be exposed to sexual practices. ${ }^{44}$ Besides, adolescents who are not attending school are left without access to comprehensive sexual education, skills needed to negotiate sexuality and reproductive options to prevent pregnancy. ${ }^{6}$

Evidence has shown that educational level is associated with teenage pregnancy. ${ }^{10,45}$ The current study also found teenage pregnancy is significantly related to teenagers with no formal education. This finding is in accordance with a review done in European Union countries by Imamura et $\mathrm{al}^{46}$ and a study conducted in East African countries. ${ }^{47}$ Education helps the adolescent to attain better knowledge about sexual and reproductive health, including fertility and conception. ${ }^{48}$ Moreover, access to education is relevant to girls to be able to negotiate reproductive desires, including when and how many children to have. ${ }^{6}$ Uneducated women are less empowered than educated women to negotiate with their partners about when to practice sex and are more prone to encounter genderbased violence and marry earlier. ${ }^{49}$

Despite legislative efforts to prevent child marriage, the practice persists across several Sub-Saharan African countries. ${ }^{50}$ This study showed child marriage was associated with teenage pregnancy which agrees with studies carried out in African countries including Ethiopia, ${ }^{40}$ Nigeria, ${ }^{51}$ and Uganda. ${ }^{52}$ In early marriage, a teenager is exposed to frequent and unprotected sexual activity, often leading to an early and risky first birth. A study from Nicaragua discussed child marriage as a risk for adolescent pregnancy due to the strong expectations that fertility was proved by conceiving within a year of marriage. ${ }^{53}$

It was found that teenagers from divorced parents were more exposed to pregnancy than those from married parents. This finding is in line with studies conducted in Northeast Ethiopia, ${ }^{29}$ Malaysia, $^{54}$ and South Africa, ${ }^{55}$ which reported, in comparison to teenagers from married parents, teenagers from divorced parents were more prone to teenage pregnancy. The attitude of children on sexual behaviors changed due to their parental divorce, ${ }^{56}$ children whose parents lived together had less chance of early sexual practice than those whose parents were divorced or separated. ${ }^{57}$ Furthermore, children delay sexual practice when there is good communication regarding sexual education with both parents compared to just a single one. ${ }^{58}$ Literature also points out that children of divorced parents are more likely to take on adults' roles, such as early marriage. An early marriage can grant the break out of an unpleasant family situation or an emotional replacement missing in the parental home. ${ }^{59}$

Having an elder sister who was pregnant was considered a critical life experience for becoming a teen mother, ${ }^{23}$ consistent with other studies conducted by Soontrapirom et al in Thailand, ${ }^{22}$ Wall-Wieler et al in Canada, ${ }^{60}$ and East et al in the USA. ${ }^{61}$ Our study also demonstrated that there was a significant association between teenage pregnancy and having a sister who had a history of teenage pregnancy. Family members contribute to both an individuals' attitudes and values 
concerning teenage pregnancy; family shares social risks that influence the likelihood of teenage pregnancy. According to social learning and modeling theories, younger sisters are more likely to become pregnant if their older sisters were adolescent mothers. ${ }^{60}$

The awareness of fertility increases the ability to identify the associated risks of sexual behavior, and decisionmaking concerning childbearing. Lack of knowledge of fertility leads to multiple undesirable health outcomes, such as unintentional or unplanned pregnancy in a marriage, and unsafe abortion among unmarried adolescences. $^{62,63}$ The finding of this study revealed that the prevalence ratio of teenage pregnancy was 1.3 times higher among teenagers who did not know the fertile time during the menstrual cycle compared to teenagers who knew the fertile time. This is consistent with other related studies in Ethiopia ${ }^{64}$ and African countries. ${ }^{65}$ The studies showed that knowledge about the ovulatory cycle reduces the likelihood of becoming pregnant.

Despite the above valuable findings, this study has its own limitations. Responses to the outcome and independent variable measurements may be affected by social desirability bias. For that matter, asking about pregnancy, especially among unmarried teenagers, is considered a cultural taboo in the study area. To this end, the following measures were taken to reduce the social desirability bias: the interview was conducted only by experienced female data collectors in private places and selected familiar sexual terms that take the culture of the community into account were used.

\section{Conclusion}

One in three teenagers had been pregnant in Kersa at the time of the survey. This indicates that teenage pregnancy was prevalent in the study area. Increased age, not being in school, having lack of formal education, being married, parental divorce, having an elder sister who had a history of teenage pregnancy, and not knowing the fertile period in menstrual cycles were all significantly associated with teenage pregnancy. The findings highlighted the need to strengthen policy issues to focus on programs, such as preventing child marriage, retaining teenagers in school, educating teenage girls, and creating awareness on reproductive health issues.

\section{Abbreviations}

APR, adjusted prevalence ratio; CI, confidence Interval; CPR, crude prevalence ratio; EC, emergency contraceptive; HDSS, health and demographic surveillance system; MMR, maternal mortality ratio; $\mathrm{PR}$, prevalence ratio; $\mathrm{RH}$, reproductive health; TP, teenage pregnancy; WHO, World Health Organization.

\section{Data Sharing Statement}

All the analyzed data for this article are available from the corresponding author upon reasonable request.

\section{Acknowledgment}

We would like to acknowledge Haramaya University for financial and technical support. We would also like to extend our gratitude to all the study respondents for their voluntary participation.

\section{Author Contributions}

All authors made substantial contributions to conception and design, acquisition of data, or analysis and interpretation of data; took part in the drafting of the article or revising it critically for important intellectual content; gave final approval of the version to be published; and agreed to be accountable for all aspects of the work.

\section{Disclosure}

In this work, no conflict of interest was declared by the authors.

\section{References}

1. United Nations Children's Fund, UNICEF Malaysia. World population day: young people and family planning - teenage pregnancy; 2008. https://www.unicef.org/malaysia/Teenage_ Pregnancies_-_Overview.pdf. Accessed July 2, 2020.

2. Whitehead E. Understanding the association between teenage pregnancy and inter-generational factors: a comparative and analytical study. Midwifery. 2009;25(2):147-154. doi:10.1016/j. midw.2007.02.004

3. WHO. Adolescent pregnancy; 2019. Avaliable from: https://apps.who. int/iris/bitstream/handle/10665/329883/WHO-RHR19.15eng.pdf?ua= 1. Accessed February 10, 2021.

4. Darroch J, Woog V, Bankola A, Ashford L. Adding it up: costs and benefits of meeting the contraceptive needs of adolescents. New York: Guttmacher Institute. 2016. Available from: https://www.guttmacher. org/report/adding-it-meeting-contraceptive-needs-of-adolescents.

Accessed February 10, 2021.

5. Odimegwu C, Mkwananzi S. Factors associated with teen pregnancy in sub-Saharan Africa: a multi-country cross-sectional study. Afr J Reprod Health. 2016;20(3):94-107. doi:10.29063/ajrh2016/v20i3.14

6. Loaiza E, Liang M Adolescent pregnancy: a review of evidence. New York: United Nations Population Fund; 2013. Available from: https://www.unfpa.org/sites/default/files/pub-pdf/ADOLESCENT\% 20PREGNANCY_UNFPA.pdf. Acsessed July 19, 2020.

7. Kurth F, Belard S, Mombo-Ngoma G, et al. Adolescence as risk factor for adverse pregnancy outcome in Central Africa-a cross-sectional study. PLoS One. 2010;5(12):e14367. doi:10.1371/journal. pone. 0014367 
8. Mersal FA, Esmat OM, Khalil GM. Effect of prenatal counselling on compliance and outcomes of teenage pregnancy. East Mediterr Health J. 2013;19(1):10-17. doi:10.26719/2013.19.1.10

9. Eyasu AM. Determinants of adolescent fertility among Rural Women of Ethiopia. OALib. 2016;03(03):1-9. doi:10.4236/oalib.1102422

10. Chen $\mathrm{CK}$, Williams K. Investigating risk factors affecting teenage pregnancy rates in the United States. Eur Int J Sci Technol. 2013;2 (2).

11. WHO. Trends in Maternal Mortality 2000 to 2017: Estimates by WHO, UNICEF, UNFPA, World Bank Group and the United Nations Population Division. Geneva: World Health Organization; 2019.

12. UN. Sustainable development goal 3: ensure healthy lives and promote well-being for all at all ages: United Nation; 2017. Available from: http://www.un.org/sustainabledevelopment/health/. Accessed February 10, 2021.

13. Kalipeni E, Iwelunmor J, Grigsby-Toussaint D. Maternal and child health in Africa for sustainable development goals beyond 2015 Glob Public Health. 2017;12(6):643-647. doi:10.1080/ 17441692.2017.1304622

14. Moni SA, Nair MK, Devi RS. Pregnancy among unmarried adolescents and young adults. J Obstet Gynaecol India. 2013;63(1):49-54. doi:10.1007/s13224-012-0244-7

15. UN Population Fund (UNFPA). Motherhood in Childhood: facing the challenge of adolescent pregnancy; 2013. Available from: https:// www.refworld.org/docid/52fcc7e84.html. Accessed December 11, 2020.

16. Akella D, Jordan M. Impact of social and cultural factors on teen pregnancy. J Health Dispar Res Pract. 2015;8(1):41-62.

17. Suan MAM, Ismail AH, Ghazali H. A review of teenage pregnancy research in Malaysia. Med $J$ Malaysia. 2015;70 (4):214-219.

18. United Nations, Department of Economic and Social Affairs, Population Division Adolescent Fertility since the International Conference on Population and Development (ICPD) in Cairo (United Nations publication). 2013

19. Gökçe B, Özşahin A, Zencir M. Determinants of adolescent pregnancy in an urban area in Turkey: a population-based case-control study. $J$ Biosoc Sci. 2007;39(2):301-311. doi:10.1017/ S0021932006001763

20. Kassa GM, Arowojolu AO, Odukogbe AA, Yalew AW. Prevalence and determinants of adolescent pregnancy in Africa: a systematic review and meta-analysis. Reprod Health. 2018;15(1):195. doi:10.1186/s12978-018-0640-2

21. Garwood SK, Lara G, Melissa JR, Katie P, Brett D. More than poverty - teen pregnancy risk and reports of child abuse reports and neglect. $J$ Adolesc Health. 2015;57(2):164-168. doi:10.1016/j. jadohealth.2015.05.004.

22. Soontrapirom T, Tripathi S, Talungchit $\mathrm{P}$, Apinuntavech $\mathrm{S}$. Life assets in teenage pregnancy. Siriraj Med J. 2017;69(3). doi:10.14456/ smj.2017.24

23. Dangal G. Teenage Pregnancy: complexities and Challenges. $J$ Nep Med Assoc. 2006;45:262-272. doi:10.31729/jnma.513

24. Pati RN, Tekie S. Biocultural dynamics of teenage pregnancies in Ethiopia: medico anthropological appraisal. Int J Soc Sci Manag. 2016;3(1):68-77. doi:10.3126/ijssm.v3i1.14368

25. Central Statistical Agency (CSA) [Ethiopia] and ICF. Ethiopia Demographic and Health Survey 2016. Addis Ababa, Ethiopia, and Rockville, Maryland, USA: CSA and ICF; 2016.

26. Taffa N, Obare F. Pregnancy and child health outcomes among adolescents in Ethiopia. Ethiop J Health Dev. 2004;18(2):90-95.

27. Assefa B, Abiyou M, Yeneneh G, et al. Assessment of the magnitude of teenage pregnancy and its associated factors among teenage females visiting Assosa General Hospital. Ethiop Med J. 2015; (Supp. 2):25-37.
28. Mathewos S, Mekuria A. Teenage pregnancy and its associated factors among school adolescents of Arba Minch Town, Southern Ethiopia. Ethiop J Health Sci. 2018;28(3):287-298. doi:10.4314/ejhs. v28i3.6

29. Habitu YA, Yalew A, Bisetegn TA. Prevalence and factors associated with teenage pregnancy, Northeast Ethiopia, 2017: a cross-sectional study. J Pregnancy. 2018. doi:10.1155/2018/1714527

30. Assefa N, Oljira L, Baraki N, et al. HDSS Profile: The Kersa Health and Demographic Surveillance System. Int J Epidemiol. 2016;45 (1):94-101. doi:10.1093/ije/dyv284

31. WHO. Asking young people about sexual and reproductive behaviors: illustrative questionnaire for interview-surveys with young people [Internet]. John Clevland: Geneva. Available from: https:// www.who.int/reproductivehealth/topics/adolescence/questionnaire. pdf?ua $=1 \mathrm{en} /$. Accessed February 10, 2021.

32. Barros AJ, Hirakata VN. Alternatives for logistic regression in cross-sectional studies: an empirical comparison of models that directly estimate the prevalence ratio. BMC Med Res Methodol. 2003;3(1). doi:10.1186/1471-2288-3-21

33. Martinez BAF, Leotti VB, Silva GSE, Nunes LN, Machado G, Corbellini LG. Odds ratio or prevalence ratio? An overview of reported statistical methods and appropriateness of interpretations in cross-sectional studies with dichotomous outcomes in veterinary medicine. Front Vet Sci. 2017;4:193. doi:10.3389/ fvets. 2017.00193

34. Okereke CI. Assessing the prevalence and determinants of adolescents' unintended pregnancy and induced abortion in Owerri, Nigeria. J Biosoc Sci. 2010;42(5):619-632. doi:10.1017/S0021932010000179

35. Nwosu UM. Contemporary factors of teenage pregnancy in rural communities of Abia state, Nigeria. Int J Community Med Public Health. 2017;4(2):588-592. doi:10.18203/2394-6040. ijcmph20170295

36. Islam AZ, Mondal MN, Khatun ML, et al. Prevalence and determinants of contraceptive use among employed and unemployed women in Bangladesh. Int J MCH AIDS. 2016;5(2):92-102. doi:10.21106/ ijma. 83

37. Todd N, Black A. Contraception for adolescents. J Clin Res Pediatr Endocrinol. 2020;12(Suppl 1):28-40. doi:10.4274/jcrpe.galenos.2019.2019.S0003

38. Alemayehu T, Haider J, Habte D. Determinants of adolescent fertility in Ethiopia. Ethiop J Health Dev. 2010;24(1):30-38.

39. Were M. Determinants of teenage pregnancies: the case of Busia District in Kenya. Econ Hum Biol. 2007;5(2):322-339. doi:10.1016/ j.ehb.2007.03.005

40. Ayele BG, Gebregzabher TG, Hailu TT, Assefa BA, van Wouwe JP. Determinants of teenage pregnancy in Degua Tembien District, Tigray, Northern Ethiopia: a community-based case-control study. PLoS One. 2018;13(7):e0200898. doi:10.1371/journal.pone.0200898

41. Rosenberg M, Pettifor A, Miller WC, et al. Relationship between school dropout and teen pregnancy among rural South African young women. Int $J$ Epidemiol. 2015;44(3):928-936. doi:10.1093/ije/ dyv007

42. Glynn JR, Sunny BS, Destavola B, et al. Early school failure predicts teenage pregnancy and marriage: a large population-based cohort study in northern Malawi. PLoS One. 2018;13(5):e0196041. doi:10.1371/journal.pone.0196041

43. Jukes M, Simmons S, Bundy D. Education and vulnerability: the role of schools in protecting young women and girls from HIV in southern Africa. AIDS. 2008;22(Suppl 4):S41-56. doi:10.1097/01. aids.0000341776.71253.04

44. Barnes GM, Hoffman JH, Welte JW, Farrell MP, Dintcheff BA. Adolescents' time use: effects on substance use, delinquency and sexual activity. J Youth Adolesc. 2007;36:697-710. doi:10.1007/ s10964-006-9075-0 
45. Raj AD, Rabi B, Amudha P, Edwin VT, Glyn C. Factors associated with teenage pregnancy in South Asia: a systematic review. Health Sci J. 2010;4(1):3-14.

46. Imamura M, Tucker J, Hannaford P, et al. Factors associated with teenage pregnancy in the European Union countries: a systematic review. Eur J Public Health. 2007;17(6):630-636. doi:10.1093/eur$\mathrm{pub} / \mathrm{ckm} 014$

47. Neal SE, Chandra-Mouli V, Chou D. Adolescent first births in East Africa: disaggregating characteristics, trends and determinants. Reprod Health. 2015;12(1). doi:10.1186/1742-4755-12-13

48. Dulitha F, Nalika G, Upul S, et al. Risk factors for teenage pregnancies in Sri Lanka: perspective of a community based study. Health Sci J. 2013;7(3):269-284.

49. Ayele WM. Differentials of early teenage pregnancy in Ethiopia, 2000, and 2005. PLoS One. 2013;90.

50. Yaya S, Odusina EK, Bishwajit G. Prevalence of child marriage and its impact on fertility outcomes in 34 sub-Saharan African countries. BMC Int Health Hum Rights. 2019;19(1):33. doi:10.1186/s12914019-0219-1

51. Envuladu EA, Agbo HA, Ohize VA, Zoakah AI. Determinants and outcome of teenage pregnancy in a rural community in Jos, Plateau State, Nigeria. Sub-Saharan Afr J Med. 2014;1(1):48-52. doi: $10.4103 / 2384-5147.129319$

52. Gideon R. Factors associated with adolescent pregnancy and fertility in Uganda: analysis of the 2011 demographic and health survey data. Soc Sci. 2013;2(1):7-13. doi:10.11648/j.ss.20130201.12

53. Lion KC, Prata N, Stewart C. Adolescent childbearing in Nicaragua: a quantitative assessment of associated factors. Int Perspect Sex Reprod Health. 2009;35(2):91-96. doi:10.1363/3509109

54. Abdullah S, Ghani SA, Sipon S, Akil SM, Faudzi NM. Relationship of parent and peer attachment with coping strategy among teenagers pregnancy. Procedia Soc Behav Sci. 2014;114:334-338. doi:10.1016/ j.sbspro.2013.12.707

55. Odimegwu C, Mkwananzi S. Family structure and community connectedness: their association with teenage pregnancy in South Africa. $J$ Psychol Afr. 2018;28(6):479-484. doi:10.1080/ 14330237.2018 .1544390
56. Jo Ânsson FH, Njaròvik U, Oâlafsdo Âttir G, Greâ Tarsson SJ. Parental divorce: long-term effects on mental health, family relations and adult sexual behavior. Scand J Psychol. 2000;41(2):101-105. doi:10.1111/1467-9450.00177

57. Salih NA, Metaferia H, Reda AA, Biadgilign S. Premarital sexual activity among unmarried adolescents in northern Ethiopia: a cross-sectional study. Sex Reprod Healthc. 2015;6(1):9-13. doi:10.1016/j.srhc.2014.06.004

58. Stammers T. Teenage pregnancies are influenced by family structure. BMJ. 2002;324(7328):51. doi:10.1136/bmj.324.7328.51a

59. Martin V, Mills M, Bourdais CL. The consequences of parental divorce on the life course outcomes of Canadian children. Can Stud Popul. 2005;32(1):29-51. doi:10.25336/P6V315

60. Wall-Wieler E, Roos LL, Nickel NC. Teenage pregnancy: the impact of maternal adolescent childbearing and older sister's teenage pregnancy on a younger sister. BMC Pregnancy Childbirth. 2016;16 (1):120. doi:10.1186/s12884-016-0911-2

61. East PL, Reyes BT, Horn EJ. Association between adolescent pregnancy and a family history of teenage births. Perspect Sex Reprod Health. 2007;39(2):108-115. doi:10.1363/3910807

62. Thijssen A, Meier A, Panis K, Ombelet W. Fertility awareness-based methods' and subfertility: a systema tic review. Facts Views Vis Obgyn. 2014;6(3):113-123.

63. Hampton K, Mazza D. Fertility-awareness knowledge, attitudes and practices of women attending general practice. Aust Fam Physician. 2015;44(11):840-845.

64. Geda YF. Determinants of teenage pregnancy in Ethiopia: a case-control study, 2019. Curr Med Issues. 2019;17(4):112. doi:10.4103/cmi.cmi_12_19

65. Iyanda AE, Dinkins BJ, Osayomi T, Adeusi TJ, Lu Y, Oppong JR. Fertility knowledge, contraceptive use and unintentional pregnancy in 29 African countries: a cross-sectional study. Int J Public Health. 2020;65(4):445-455. doi:10.1007/s00038-020-01356-9
International Journal of Women's Health

\section{Publish your work in this journal}

The International Journal of Women's Health is an international, peerreviewed open-access journal publishing original research, reports, editorials, reviews and commentaries on all aspects of women's healthcare including gynecology, obstetrics, and breast cancer. The manuscript management system is completely online and includes a very quick and fair peer-review system, which is all easy to use. Visit http://www.dovepress.com/testimonials.php to read real quotes from published authors. 